\title{
Spin-polarized free electron beam interaction with radiation and superradiant spin-flip radiative emission
}

\author{
A. Gover \\ Faculty of Engineering, Department of Physical Electronics, Tel-Aviv University, Israel
}

(Received 28 February 2006; published 13 June 2006)

\begin{abstract}
The problems of spin-polarized free-electron beam interaction with electromagnetic wave at electronspin resonance conditions in a magnetic field and of superradiant spin-flip radiative emission are analyzed in the framework of a comprehensive classical model. The spontaneous emission of spin-flip radiation from electron beams is very weak. We show that the detectivity of electron spin resonant spin-flip and combined spin-flip/cyclotron-resonance-emission radiation can be substantially enhanced by operating with ultrashort spin-polarized electron beam bunches under conditions of superradiant (coherent) emission. The proposed radiative spin-state modulation and the spin-flip radiative emission schemes can be used for control and noninvasive diagnostics of polarized electron/positron beams. Such schemes are of relevance in important scattering experiments off nucleons in nuclear physics and off magnetic targets in condensed matter physics.
\end{abstract}

DOI: 10.1103/PhysRevSTAB.9.060703

PACS numbers: $29.27 . \mathrm{Hj}, 34.80 . \mathrm{Nz}, 41.60 . \mathrm{Cr}$

\section{INTRODUCTION}

All electron beam radiation sources, in a spectral range stretching from the microwave to $\mathrm{x}$-rays, are based on interaction of the free-electron charge with the electric field of an electromagnetic (EM) wave. These include spontaneous emission schemes as Cerenkov radiation and synchrotron radiation, as well as stimulated emission schemes as microwave tubes and free-electron laser (FEL) $[1,2]$.

Electrons (as well as positrons and other particles) are also endowed with magnetic moment due to their spin, and can emit radiation or exchange energy with an EM wave by interaction with its magnetic field. This interaction is weak relative to the electric interaction, and magnetic radiative emission has never been observed from free particle beams. However, there is no fundamental restriction for its observation. In fact, in a solid, where the density of bound electrons is high, several spin-transition radiation devices have been demonstrated: the first masers were based on stimulated emission of bound electrons at spin resonance condition in a magnetic field [3]; and electron spin resonance (ESR), electron paramagnetic resonance (EPR), and nuclear magnetic resonance (NMR) are well known effects of interaction between an EM radiation wave and the magnetic moments of bound electrons or atomic nuclei in condensed matter. To the best knowledge of the author, resonant ESR spin-flip emission has never been observed in free electrons. However, the occurrence of spontaneous spin-flip radiative emission by free electrons is evidenced indirectly in synchrotron storage rings. It is manifested when electrons (or positrons) are subjected to traverse magnetic forces in the ring. At steady state the cumulative effect of the emissions polarizes (transversely) the spin state of the circulating charged particles beam [4,5]. The excess (nonresonant) spin-flip synchrotron spon- taneous emission was also directly measured in an intricate experiment in a storage ring [6] and in an experiment of hard x-ray generation by an energetic electron beam incident at shallow angle on a single crystal [7].

The goal of the present paper is to find the characteristics and the conditions for observation of ESR free-electron spin-flip emission of radiation (FESFER) and in particular its enhancement by the process of superradiance (SR) [812]. We also solve the equations of interaction (stimulated emission/absorption) between a radiation wave and a polarized-beam: free-electron spin modulation by electromagnetic radiation (FESMER). This process is useful for preparing the electron beam magnetic polarization state for maximal FESFER emission. It is also of independent interest in other experimental schemes, when there is need to control the polarization state of a polarized electron beam. This is required in experiments of scattering of a polarized electron beam off spin-polarized targets. In such experiments information is derived from the polarization-state dependent scattering cross section. In such experiments the radiative interaction may serve both for noninvasive diagnostics and for control of the $e$-beam polarization state.

Recent progress in development of spin-polarized photocathode $e$-gun injectors [13] for RF-LINAC accelerators led to important measurements and discoveries in nuclear physics (e.g., $[14,15])$. The electron beams, produced in such photocathode $e$-guns, are usually made of picosecond bunches, corresponding to the pulse duration of the pump lasers, which generate them, by virtue of the photoelectric effect, out of an illuminated semiconductor cathode. The electrons emitted from the crystalline semiconductor have distinct quantum spin states $( \pm 1 / 2)$ relative to the axial (emission) direction, and a net polarization level $\left(P_{\uparrow}-\right.$ $\left.P_{\downarrow}\right) /\left(P_{\uparrow}+P_{\downarrow}\right)$ as high as $80 \%$ may be achieved [13]. The axial polarization of the spins can be rotated and trans- 
formed to transverse polarization by a combination of static magnetic and electric field deflectors. It can also be done in a FESMER section, as proposed in this paper, by employing an external electromagnetic wave at ESR condition. This can then be carried out without diverting the axial propagation transport line of the beam.

It is shown here that the superradiant radiation pulses that are emitted by such transversely polarized $e$-beam bunches, upon traversal through an axial magnetic field, can be observable, and possibly can be used to indicate the polarization level of the electron beam. It is also suggested that FESMER controlled spin-polarized low energy $e$-beams can be useful for extending already existing technologies for diagnostics of magnetic polarization and spin-states in solids [16-18] and may contribute to the development of the new evolving technological field of "Spintronics" [19]. With these observations, one may expect, that in addition to its fundamental interest, the complete analysis of FESFER and FESMER processes, to be presented here, will have significance in the advancement of important applications of spin-polarized beams.

Exposing in advance the limitations of the classical model used here, we recognize that the spin is a quantum-mechanical entity and when inserted in a magnetic field, it constitutes in its rest frame a system of distinct two-quantum states. Nevertheless, in the next two sections we use a classical approach to analyze the FESMER process, which involves nonlinear interaction (stimulated emission/absorption) of the spins with an intense radiation field. This is well justified (and particularly so when the e-beam is polarized), as is commonly done in ESR, NMR, and conventional two-level laser systems $[3,20]$. In the subsequent sections we analyze the spontaneous and superradiant emission processes also on the basis of a classical model. This is again justifiable for the superradiant case as was demonstrated in the treatise of Dicke (who starts from quantum electrodynamics analysis and obtains the classical results in the limit of a large number of coherently emitting dipoles) [8]. However, the classical analysis is not valid, for the calculation of spontaneous emission, where the emission of a photon by a single electron involves reversal of its spin state and where quantization of the radiation field needs to be included. Thus the calculation of spontaneous emission in these sections is valuable primarily as a step for calculating the phenomenon of interest of enhanced superradiant emission [21]. For a rigorous calculation of the spontaneous emission, a quantum analysis as in [4,5,22] is needed. A brief discussion, following the formulation of Dicke, on the transition from the quantum to classical limit in the case of superradiance, is given in Appendix A. Plausibility arguments in Appendices A and B make it possible to confirm the derived classical FESFER expressions and obtain the necessary quantum mechanical modifications to the spontaneous emission expressions.

\section{ESR RADIATION CONDITIONS OF AN ELECTRON BEAM}

The classical magnetic moment of a particle with spin $\mathbf{S}$, which is subjected to magnetic field $B^{\prime}$ (in its relativistic rest frame), is governed by

$$
\begin{gathered}
\frac{d \boldsymbol{\mu}^{\prime}}{d t}=\boldsymbol{\mu}^{\prime} \times \boldsymbol{\omega}_{s}^{\prime}, \\
\boldsymbol{\omega}_{s}^{\prime}=\frac{g e}{2 m} \mathbf{B}^{\prime}, \\
\left|\boldsymbol{\mu}^{\prime}\right|=\frac{\hbar g e}{2 m} S=\mu_{B} g S=\mu_{s}, \\
\Delta E^{\prime}=2 \mu_{s} B^{\prime}=\frac{\hbar g e}{m} S B^{\prime}=\hbar \omega_{s}^{\prime} .
\end{gathered}
$$

Here $\Delta E^{\prime}$ is the maximum spin-flip energy, $g=$ 2.0023193 is the Lande $g$ factor [23,24], and $\mu_{B}=$ $\hbar e / 2 m$ is the Bohr magneton. For an electron or a positron $S=1 / 2$ and $\mu_{s}=9.2848 \times 10^{-24} \mathrm{~J} / \mathrm{T}$. SI units system is being used throughout. It is pointed out that rigorously Eq. (1) is defined only for the spin operator $\mathbf{S}$ (and is valid even for $g=0$ ). In our classical model we apply it to the magnetic moment $\boldsymbol{\mu}^{\prime}$, using the connection (3). This is appropriate for particles like electrons for which $g \cong 2$. It should be born in mind that the spin $\mathbf{S}$ and the magnetic moment $\boldsymbol{\mu}^{\prime}$ are defined only in the electronic rest frame. For this reason we keep the prime sign all along.

For an $e$-beam propagating on an axis $(z)$ parallel to a uniform magnetic field $\mathbf{B}=B_{0} \hat{\mathbf{e}}_{z}$ with velocity $\boldsymbol{\beta}=$ $\beta \hat{e}_{z}$ and energy $\gamma m c^{2}$, the electron spin resonance (ESR) frequency in the particle rest frame is (note: $B_{z}=B_{z}^{\prime}$ )

$$
\omega_{s 0}^{\prime}=\frac{g e}{2 m} B_{0} .
$$

Assuming negligible quantum recoil effect, the electron spin, precessing at frequency $\omega_{s 0}^{\prime}$, can emit or interact with an electromagnetic (EM) wave of the same frequency $\omega_{s 0}^{\prime}$ (in its relativistic frame). Consider an EM wave (a waveguide mode or a free space plane wave) emitted or interacting with the spin at its ESR frequency $\omega_{s 0}^{\prime}$ :

$$
\begin{aligned}
& \mathbf{E}^{\prime}\left(\mathbf{r}^{\prime}, t^{\prime}\right)=\operatorname{Re}\left\{\tilde{\mathbf{E}}\left(\mathbf{r}^{\prime}\right) e^{i k_{z o}^{\prime} z-i \omega_{s o}^{\prime} t}\right\}, \\
& \mathbf{B}^{\prime}\left(\mathbf{r}^{\prime}, t^{\prime}\right)=\operatorname{Re}\left\{\tilde{\mathbf{B}}\left(\mathbf{r}_{\perp}^{\prime}\right) e^{i k_{z o}^{\prime} z^{\prime}-i \omega_{s o}^{\prime} t^{\prime}}\right\} .
\end{aligned}
$$

The frequency of this wave will be seen in the laboratory frame as

$$
\omega_{r}(\Theta)=\frac{\omega_{s o}^{\prime} / \gamma}{1-\beta \cos \Theta},
$$

where $\cos \Theta=k_{z} / k\left(k=\omega_{r} / c\right)$. For $\Theta=0$ (forward emission of a TEM wave), the radiation is Doppler-upshifted to

$$
\omega_{r 0}=\omega_{r}(0)=(1+\beta) \gamma \omega_{s 0}^{\prime} .
$$


With commonly available magnetic fields $-\quad 0.5 \mathrm{~T}$ (for normal magnets) or $10 \mathrm{~T}$ (with superconducting magnets) - the rest-frame ESR frequencies are in the microwave up to terahertz regime: $f_{s 0}^{\prime}=50 \mathrm{GHz}-1 \mathrm{THz}$. With moderate acceleration to $E=100 \mathrm{MeV}$, the forward FESFER emission in the laboratory frame [Eq. (8)] can be, for these magnetic fields, in the IR-up to visible regime $\left(\lambda_{r 0}=15-0.75 \mu \mathrm{m}\right)$.

The maximum FESFER radiative power that can be extracted from a fully polarized upper level excited electron beam of average current $I$ is

$$
P=\hbar \omega_{r 0}(0) I / e,
$$

which for the latter example $\left(\lambda_{r 0}=0.75 \mu\right)$ corresponds to $1.65 \mathrm{~W} / \mathrm{A}$. Note, however, that this much radiative power is available only if all electrons are induced to radiate in the forward direction, and all of them make full transition to the lower spin level. When considering the average pulse power emission from a single e-beam bunch, the average current $I$ is calculated as the bunch charge, divided by the radiation pulse duration [the bunch pulse duration plus the "slippage time" of the radiation, which for forward emission of a free space radiation wave is given by $(1-$ $\beta) L / \beta c]$.

Equation (9) also gives the radiation power that would be absorbed from a radiation wave by a spin-polarized $e$-beam making full transition from the low energy (spin down) state to the higher energy (spin up) state (a FESMER process).

Figure 1 displays a free-electron spin-flip radiative emission scheme that incorporates the two processes of ESR interaction between a polarized $e$-beam and a radiative wave:

(i) A FESMER process of spin-polarization-state rotation takes place in the spin modulation section. This process can be described as stimulated absorption of the modulating radiation wave, which changes the polarization

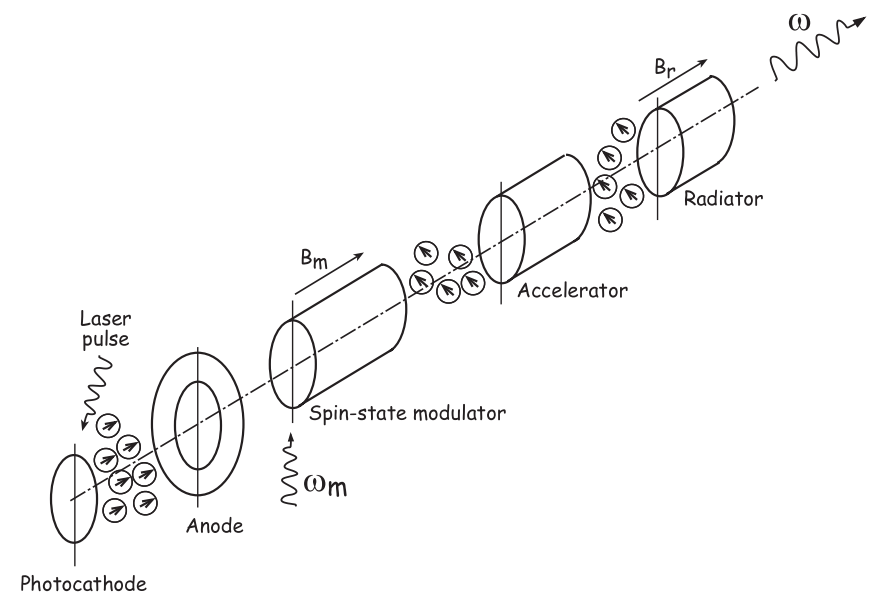

FIG. 1. A scheme for electron spin-state rotation (FESMER) and radiation (FESFER). state of the electron beam from longitudinal to transverse polarization or to any other polarization state.

(ii) A process of FESFER takes place in the radiating section. This process is most intense when it is superradiant, namely, when the electron beam is transversely polarized (has maximal magnetic dipole moment in the transverse dimension) and is either axially bunched at the radiative emission frequency $f_{r}=\omega_{r} / 2 \pi$, or has a pulse duration $t_{p}$ short relative to this frequency $f_{r} t_{p} \ll 1$. In these cases all the electron spins precess and emit in phase, forming a giant magnetic dipole moment. The bunched electron beam emits then an intense superradiant radiation wave $[9,10]$.

The experimentally verified fact that out of the magnetic sections, and even in the acceleration stage, the electrons keep their spin state, makes it possible to perform the two processes at two different frequencies, and produce in effect (for forward emission) a wave frequency transformer of frequency up-shift ratio [see Eq. (7)]:

$$
\frac{\omega_{r}}{\omega_{m}}=\frac{\gamma_{m}\left(1-\beta_{m} \cos \Theta_{m}\right)}{\gamma_{r}\left(1-\beta_{r} \cos \Theta_{r}\right)} \frac{B_{r}}{B_{m}},
$$

where $m$ and $r$ correspond to the modulation and radiation sections, respectively. For example, if the modulation is done at $E_{m}=100 \mathrm{keV}, \Theta_{m} \cong \pi / 2$, and the radiation at $E_{r}=100 \mathrm{MeV}, \Theta_{r} \cong 0$, and $B_{r}=B_{m}$, the frequency upshift factor is $\times 286$.

\section{STIMULATED RADIATIVE INTERACTION, SPIN-STATE MODULATION}

To explore the interaction between a radiation wave and the electrons spin it is convenient to use the Bargmann, Michel, Telegdi (BMT) equation [24], which describes the dynamics of the electron rest-frame spin $\mathbf{S}$ in terms of labframe coordinates and fields:

$$
\begin{aligned}
\frac{d \mathbf{S}}{d t}= & \frac{e}{m \gamma} \mathbf{S} \times\left[(1+\gamma a) \mathbf{B}-\frac{a \gamma^{2}}{\gamma+1} \boldsymbol{\beta}(\boldsymbol{\beta} \cdot \mathbf{B})\right. \\
& \left.-\left(a+\frac{1}{\gamma+1}\right) \frac{\gamma}{c} \boldsymbol{\beta} \times \mathbf{E}\right],
\end{aligned}
$$

where $a=(g-1) / 2=0.00115965$. We apply this, in our classical model, to the magnetic moment $\boldsymbol{\mu}^{\prime}$, and set $a=0(g \cong 2)$. Assuming the electron propagates along a uniform axial magnetic field section $B=B_{0} \hat{e}_{z}$, and interacts with a copropagating intense (classical) electromagnetic wave

$$
\{\mathbf{E}(\mathbf{r}, t), \mathbf{B}(\mathbf{r}, t)\}=\operatorname{Re}\left[\left\{\tilde{\mathcal{E}}\left(\mathbf{r}_{\perp}\right), \tilde{\mathcal{B}}\left(\mathbf{r}_{\perp}\right)\right\} e^{-i \omega_{m} t+i k_{z m} z}\right]
$$

the BMT equation can be written as

$$
\frac{d \boldsymbol{\mu}^{\prime}}{d t}=\boldsymbol{\mu}^{\prime} \times\left[\boldsymbol{\omega}_{s 0}+\boldsymbol{\Omega}_{r}(t)\right]
$$

where 


$$
\begin{gathered}
\boldsymbol{\omega}_{s 0}=\omega_{s 0} \hat{\mathbf{e}}_{z}, \quad \omega_{s 0}=\omega_{r}(\theta=\pi / 2)=\omega_{s 0}^{\prime} / \gamma, \\
\boldsymbol{\Omega}_{r}(t) \cong \frac{e}{m \gamma}\left(1-\frac{\gamma \beta}{\gamma+1} \cos \Theta_{m}\right) \mathbf{B}_{\perp}(\mathbf{r}, t)
\end{gathered}
$$

[here we assumed for simplicity, a specific case where the radiation wave (11) is a TEM or TM wave and having no axial magnetic field component].

In the absence of radiation field $\left(\Omega_{r}=0\right)$ the solution of Eq. (12) is

$$
\begin{aligned}
\boldsymbol{\mu}_{\perp}^{\prime}(t) & =\operatorname{Re}\left[\tilde{\mu}_{+}^{\prime} \hat{\mathbf{e}}_{+} e^{-i \omega_{s 0}\left(t-t_{0}\right)+i \varphi_{s 0}}\right], \\
\mu_{z}^{\prime}(t) & =\sqrt{\mu_{s}^{2}-\boldsymbol{\mu}^{\prime 2}(t)}=\sqrt{\mu_{s}^{2}-\left|\tilde{\mu}_{+}^{\prime}\right|^{2} / 2}=\mathrm{const},
\end{aligned}
$$

where $\hat{\mathbf{e}}_{ \pm} \equiv\left(\hat{\mathbf{e}}_{x} \pm i \hat{\mathbf{e}}_{y}\right) / \sqrt{2}$ and $\mu_{s}=\mu_{B} g / 2$ [Eq. (3)], $t_{0}$ is the electron entrance time into the magnetic field region and $\varphi_{s 0}$-its initial precession phase.

We now solve Eq. (12) for an electron, precessing at frequency $\omega_{s 0}$ in an axial magnetic field $B_{0}$, while the radiation field (11) is also applied. Assume that the modulation frequency $\omega_{m}$ is synchronous with the gyromagnetic frequency $\omega_{s 0}=\omega_{s 0}^{\prime} / \gamma$ at the electron position $z_{e}(t)=$ $v\left(t-t_{0}\right)$, namely [see (11)]: $\omega_{m}-k_{z}\left(\omega_{m}\right) v=\omega_{s 0}$, or $\omega_{m}=\omega_{s 0} /\left(1-\beta \cos \Theta_{m}\right)$ [in consistency with (7)]. In the "rotating vector approximation" only the right-hand polarized component of the field (11) synchronizes with the precessing field and is kept in (12) and (14):

$$
\begin{gathered}
\boldsymbol{\Omega}_{r}(t)=\operatorname{Re}\left[\tilde{\boldsymbol{\Omega}}_{r} e^{-i\left(\omega_{m}-k_{z m} v\right)\left(t-t_{0}\right)}\right], \\
\tilde{\mathbf{\Omega}}_{r}=\frac{e}{\gamma m}\left(1-\frac{\gamma \beta}{\gamma+1} \cos \Theta_{m}\right) \tilde{\mathcal{B}}_{+}\left(x_{e}, y_{e}\right) \hat{\mathbf{e}}_{+} e^{-i \omega_{m} t_{0}},
\end{gathered}
$$

where $\tilde{\mathcal{B}}_{+}\left(x_{e}, y_{e}\right)=\tilde{\mathcal{B}}\left(x_{e}, y_{e}\right) \cdot \hat{\mathbf{e}}_{-}$is the right-hand polarized field component of (11) at the electron propagation axis $\left(x_{e}, y_{e}\right)$.

The vector $\boldsymbol{\Omega}_{r}(t)$ would seem stationary in a coordinates frame rotating at frequency $\omega_{s 0}=\omega_{m}-k_{z m} v$ around the $z$ axis [20], applying a perpendicular torque moment on the spin, tending to change the polarization state from lower axial spin state $\boldsymbol{\mu}_{z}^{\prime}$ to higher, or vice versa. The solution of (12) when starting from a pure state $\mu_{z}^{\prime}\left(t_{0}\right)= \pm \mu_{s}$ is then

$$
\begin{aligned}
\mu_{z}^{\prime}(t) & = \pm \mu_{s} \cos \Omega_{\mathrm{rabi}}\left(t-t_{0}\right), \\
\left|\boldsymbol{\mu}_{\perp}^{\prime}(t)\right| & =\mu_{s} \sin \Omega_{\mathrm{rabi}}\left(t-t_{0}\right),
\end{aligned}
$$

where

$$
\begin{aligned}
\Omega_{\mathrm{rabi}} & =\left|\tilde{\mathbf{\Omega}}_{r}\right| / \sqrt{2} \\
& =\frac{e}{\sqrt{2} \gamma m}\left(1-\frac{\gamma \beta}{\gamma+1} \cos \Theta_{m}\right)\left|\hat{\mathbf{e}}_{-} \cdot \tilde{\mathcal{B}}\left(x_{e}, y_{e}\right)\right| .
\end{aligned}
$$

This spin-flip modulation process is an expression of the Rabi-oscillation between the lower and upper quantum spin levels.
The parameter of significance for the spin-state modulator is the $\pi / 2$-length $-L_{\pi / 2}$ (analogous to the $\pi / 2$-pulse duration in NMR, EPR):

$$
L_{\pi / 2}=\frac{\pi / 2}{\Omega_{\mathrm{rabi}} / v}=\frac{\pi}{\sqrt{2}}\left(\frac{1}{\beta \gamma}+\frac{\cos \Theta_{m}}{\gamma+1}\right)^{-1} \frac{m c}{e\left|\hat{\mathbf{e}}_{-} \cdot \tilde{\mathcal{B}}\right|} .
$$

At this length an axially polarized beam will turn into a transversely polarized beam, optimally prepared for highest rate of FESFER emission at the radiation section (Fig. 1).

Another parameter of merit is $L_{\pi}=2 L_{\pi / 2}$. At this length an axially polarized beam will be transformed to an axially polarized beam of the opposite sense, and a transversely polarized beam will be transformed back to a transversely polarized beam (this last process can be used to compensate for inhomogeneous broadening effects as in photon-echo effects [20]). If used in a radiation section, a power corresponding to a $\pi$-length section would correspond to the maximum emission power (9) that can be extracted by stimulated emission from an axially polarized beam.

For the example of $E_{m}=100 \mathrm{keV} B_{0}=0.5 \mathrm{~T}$, one obtains $f_{s 0}=f_{s 0}^{\prime} / \gamma=35 \mathrm{GHz}$. For a modulating radiation wave of effective cross-section area $A_{\text {em }}=10 \mathrm{~mm}^{2}$ and mode zigzag angle $\cos \Theta_{m}=0.1$ (near cutoff operation), one requires instantaneous power of $P \cong 1 \mathrm{~kW}$ to obtain $L_{\pi / 2}=1 \mathrm{~m}$.

\section{SPONTANEOUS AND SUPERRADIANT ESR FESFER}

To analyze the classical radiative emission from particulate charges and magnetic moments, we use a modal expansion of the Maxwell equations in the frequency domain:

$$
\breve{\mathbf{E}}(\mathbf{r}, \omega)=\sum_{q} \breve{C}_{q}(z) \tilde{\mathbf{E}}_{q}(\mathbf{r}), \quad \breve{\mathbf{H}}(r, \omega)=\sum_{q} \breve{C}_{q}(z) \tilde{\mathbf{H}}_{q}(\mathbf{r}),
$$

where $\quad \breve{f}(\omega)=\mathcal{F}\{f(t)\} \equiv \int_{-\infty}^{\infty} e^{i \omega t} f(t) d t, \quad$ and $\left\{\tilde{\mathbf{E}}_{q}(\mathbf{r}), \tilde{\mathbf{H}}_{q}(\mathbf{r})\right\}=\left\{\tilde{\mathcal{E}}_{q}\left(\mathbf{r}_{\perp}\right), \tilde{\mathcal{H}}_{q}\left(\mathbf{r}_{\perp}\right)\right\} e^{i k_{z q} z}$ is a set of eigenmodes of the structure (waveguide or free space) in which radiation emission takes place.

Extending the formulation of [10] to include magnetic currents, the excitation equations of the mode amplitudes is

$$
\begin{aligned}
\frac{d \breve{C}_{q}}{d z}= & -\frac{1}{4 \mathcal{P}_{q}} \iint\left[\breve{\mathbf{J}}(\mathbf{r}, \omega) \cdot \tilde{\mathbf{E}}_{q}^{*}(\mathbf{r})\right. \\
& \left.+\breve{\mathbf{J}}^{m}(\mathbf{r}, \omega) \cdot \tilde{\mathbf{H}}_{q}^{*}(\mathbf{r})\right] d^{2} r_{\perp},
\end{aligned}
$$

where $P_{q}$ is the normalization power of mode $q$. Substituting $\mathbf{J}(\mathbf{r}, t)=\sum_{j=1}^{N}-e \mathbf{v}_{j}(t) \delta\left[\mathbf{r}-\mathbf{r}_{j}(t)\right]$, $\mathbf{J}^{m}(\mathbf{r}, t)=\sum_{j=1}^{N} \dot{\boldsymbol{\mu}}_{j}^{\prime}(t) \delta\left[\mathbf{r}-\mathbf{r}_{j}(t)\right]$, one obtains 


$$
\begin{gathered}
\breve{C}_{q}(L, \omega)-\breve{C}_{q}(0, \omega)=-\frac{1}{4 \mathcal{P}_{q}} \sum_{j}\left(\Delta \breve{\mathcal{W}}_{q j}^{e}+\Delta \breve{\mathcal{W}}_{q j}^{m}\right) \\
\Delta \breve{\mathcal{W}}_{q j}^{e}=-e \int_{-\infty}^{\infty} \mathbf{v}_{j}(t) \cdot \tilde{\mathbf{E}}_{q}^{*}\left(\mathbf{r}_{j}(t)\right) d t \\
\Delta \breve{\mathcal{W}}_{q j}^{m}=\int_{-\infty}^{\infty} \dot{\boldsymbol{\mu}}_{j}^{\prime}(t) \cdot \tilde{\mathbf{H}}_{q}^{*}\left(\mathbf{r}_{j}(t)\right) d t .
\end{gathered}
$$

Setting $\breve{C}_{q}(0, \omega)=0$ (presently excluding analysis of stimulated emission), we can obtain the expression for the total spectral radiative energy emission per mode:

$$
\begin{aligned}
\frac{d W_{q}}{d \omega}= & \frac{1}{8 \pi \mathcal{P}_{q}}\left|\sum_{j=1}^{n}\left(\Delta \breve{W}_{q j}^{e}+\Delta \breve{W}_{q j}^{m}\right)\right|^{2} \\
= & \frac{1}{8 \pi \mathcal{P}_{q}}\left[\left|\sum_{j} \Delta \breve{W}_{q j}^{e}\right|^{2}+2 \operatorname{Re}\left(\sum_{j} \Delta \breve{W}_{q j}^{e} \sum_{j} \Delta \breve{\mathcal{W}}_{q j}^{m}\right)\right. \\
& \left.+\left|\sum_{j} \Delta \breve{W}_{q j}^{m}\right|^{2}\right] .
\end{aligned}
$$

Concentrating now on the pure magnetic FESFER term [third in (26)], using (11) and (15) in (25) while setting $z_{j}(t)=v\left(t-t_{0 j}\right)$ for electron $j$, one obtains an explicit expression for the average FESFER radiation spectral energy per mode $q$ :

$$
\frac{d W_{q}^{m}}{d \omega}=\frac{1}{8 \pi \mathcal{P}_{q}}\left|\Delta \breve{\mathcal{W}}_{q e}^{m}\right|^{2}\left\langle\left|\sum_{j=1}^{N} e^{i \omega_{s 0} t_{0 j}+i \varphi_{s 0 j} \sin \Psi_{j}}\right|^{2}\right\rangle_{\perp s},
$$

where $\omega_{s 0} t_{0 j}$ is the entrance phase of electron $j$, arriving to the interaction region $z=0$ at time $t_{0 j}, \varphi_{s 0 j}$ is the initial magnetic-moment precession phase at $z=0$, and $\Psi_{j}$ is the inclination angle of the magnetic-moment $\boldsymbol{\mu}_{j}^{\prime}$ relative to the $z$ axis $\left(\cos \Psi_{j}=\mu_{z}^{\prime} / \mu_{s}\right)$. This naive classical picture of the individual electron spins, which represents them essentially by the classical expectation value of their magnetic moment (3) is not the rigorous way for calculating spontaneous emission (which requires a quantum analysis $[4,5])$. Also the presentation of the magnetic current in (22) in terms of the electron rest frame parameters, requires a more accurate consideration of the relativistic transformations and Thomas precession effect, which will be reported elsewhere [22]. Nevertheless, this simplistic classical analysis is a useful step in the calculation of the classical superradiant emission, and should result in for this case quantitative estimates which would not deviate significantly from correct values.

The factor $\langle\cdots\rangle_{\perp s}$ depends on the resultant transverse magnetic moment of all the particles in a bunch. It would seem that this factor is larger, the bigger the transverse components of the individual magnetic moments, and is maximal when they are all polarized transversely $\left(\sin \Psi_{j}=1\right)$. This is a wrong conclusion when the spin gyration phases $\varphi_{s 0 j}$ or the injection phases $\omega_{s 0} t_{0 j}$ are uncorrelated. In these cases the "classical" spontaneous emission (spin shot-noise) expression (27) should be replaced by a quantum expression which has different scaling with $\Psi$ [22] (see Appendix B). However, when the beam is polarized (the gyration phases $\varphi_{0 j}$ are correlated and so are the $\Psi_{j}$ ), the large net dipole moment can be treated classically and one can proceed with Eq. (27) (see discussion in Appendix A). In this case a significant enhancement will be obtained if also the entrance times $t_{0 j}$ are correlated. If in addition to being polarized the beam will be also axially (temporally) bunched at the radiative emission frequency $f_{r}=\omega_{m} / 2 \pi$, or if its pulse duration $t_{p}$ is short relative to the optical period: $f_{r} t_{p} \ll 1$, it will radiate as a giant magnetic dipole. In this case the entire bunch would emit coherently an intense superradiant radiation pulse $[9,10]$, similar also to atomic superradiant pulse emission $[8,24]$.

Assuming all particles have the same trajectories (staying on the $z$ axis $0<z<L$ ), their common magnetic work function (25) squared is

$$
\left|\Delta \breve{W}_{q}^{m}\right|^{2}=\frac{1}{2}\left(\frac{\omega_{s 0}^{\prime} L}{\gamma v} \mu_{0} \mu_{s}\left|\tilde{\mathcal{H}}_{q+}\left(x_{e}, y_{e}\right)\right|\right)^{2} \sin c^{2}\left(\theta_{s} L / 2\right)
$$

$$
\theta_{s} L \equiv\left[\frac{\omega-\omega_{s 0}^{\prime} / \gamma}{v}-k_{z}(\omega)\right] L=2 \pi \frac{\omega-\omega_{r}(\Theta)}{\Delta \omega(\Theta)},
$$

where $\tilde{\mathcal{H}}_{q^{+}}$is the transverse right-hand circular polarization component of mode $\tilde{\mathbf{H}}_{q}$, and $\omega_{r}(\Theta)$ is given by (7). The finite interaction length homogeneous broadening linewidth of (28) at a fixed direction is

$$
\frac{\Delta \omega(\Theta)}{\omega_{r}(\Theta)}=\frac{\gamma \beta c}{f_{s 0}^{\prime} L} .
$$

The radiation pattern of (28) as function of $\Theta$ is shown in Fig. 2. It is valid for emission in free space or in a

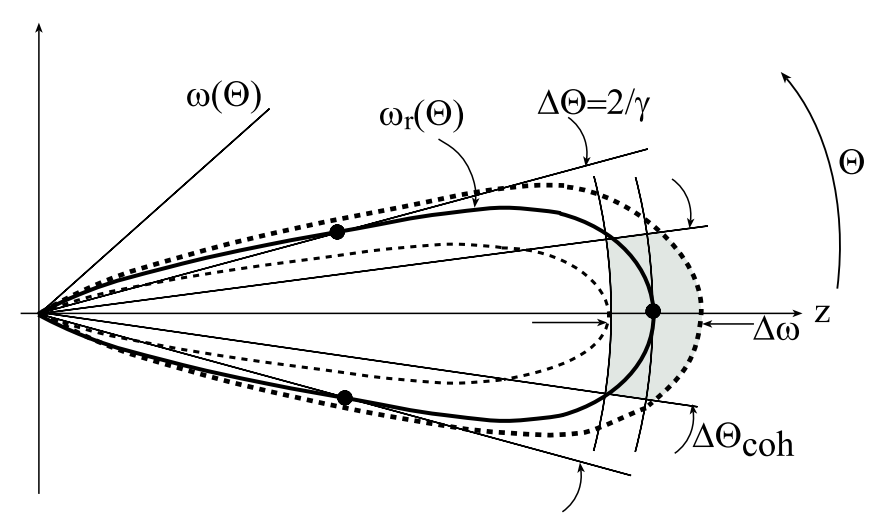

FIG. 2. The FESFER radiation pattern. The shaded section represents the phase-space region of coherent emission. 
waveguide. In a waveguide the "zigzag" angles of the radiation modes are discretized: $\cos \Theta_{q}=k_{z q}(\omega) / k=$ $\left(k^{2}-k_{c 0 q}^{2}\right)^{1 / 2} / k$ where $k_{c 0 q}$ is the cutoff wave number of mode $q$. The linewidth expression (30) is valid only when waveguide dispersion is negligible (away from zero slippage), otherwise the linewidth is wider [11]. In free space one can either use a set of discrete modes like the HermitGauss set [20] or extend the modal expansion (21) to integration over continuous radiation modes (plane waves) [25]: $\left\{\tilde{\mathcal{E}}_{q}\left(\mathbf{r}_{\perp}\right), \tilde{\mathcal{H}}_{q}\left(\mathbf{r}_{\perp}\right)\right\} \propto \exp \left(i \mathbf{k}_{\perp} \cdot \mathbf{r}_{\perp}\right)$. This would lead to an expression for the optical radiant intensity $d W / d \omega d \Omega$ [instead of (27)], which is also proportional to the same radiation pattern (28) displayed in Fig. 2.

The polar coordinates radiation pattern of Fig. 2 indicates that most of the radiation is emitted in the forward direction. For a relativistic beam most of the total emission in a wide frequency bandwidth is into a cone of $\Delta \Theta=2 / \gamma$ opening angle. The monochromatic spatially coherent radiation is emitted into a smaller cone of $\Delta \Theta_{\text {coh }}=$ $2 \sqrt{c / f_{r 0} L}=2 \sqrt{\lambda_{r 0} / \bar{L}}$ opening angle (see shaded section in Fig. 2). The number of coherent photons emission in the forward direction (emission at frequency $\left|\omega-\omega_{r 0}\right|<$ $\Delta \omega / 2)$ can be calculated then by multiplying the radiant intensity $d W / d \omega d \Omega$ by $\Delta \omega \cdot \pi\left(\Delta \Theta_{\text {coh }}\right)^{2} / 4 \hbar$, or since the radiative emission per mode is already spatially coherent, it can be simply calculated by multiplying (27) by $\Delta \omega / \hbar \omega_{r 0}$. Setting $\theta_{s}=0$ for the fundamental (free space or guided) mode $q=0$, one obtains from (27), (28), and (30):

$$
\begin{aligned}
\left(N_{\mathrm{ph}}\right)_{\mathrm{coh}} & =\frac{1}{\hbar \omega_{r 0}} \frac{d W_{0}}{d \omega} \Delta \omega=\frac{\pi}{2 \hbar} \sqrt{\frac{\mu_{0}}{\varepsilon_{0}}} \frac{\mu_{s}^{2} f_{s 0}^{\prime} L}{A_{e m} c^{3} \gamma}\langle\cdots\rangle_{\perp s} \\
& =\frac{2 \pi}{\hbar} \sqrt{\frac{\mu_{0}}{\varepsilon_{0}}} \frac{\mu_{s}^{2} f_{s 0}^{\prime 3}}{c^{5}} \frac{L}{\gamma}\langle\cdots\rangle_{\perp s} .
\end{aligned}
$$

Here we used the definition $A_{e m} \equiv \mathcal{P}_{q} / \frac{1}{2} \times$ $\sqrt{\mu_{0} / \varepsilon_{0}}\left|\tilde{\mathcal{H}}_{q+}\left(x_{e}, y_{e}\right)\right|^{2}$, and for emission in free space substituted the diffraction limited area of the fundamental Gaussian mode [10] $A_{\text {em }}=\lambda_{r 0} L / 4$. We also substituted $\beta=1$ (ultrarelativistic limit).

To calculate the total photon emission in the forward direction it is necessary to integrate $d W_{q}^{m} / d \omega / \hbar \omega$ over all frequencies and sum up over all modes $q$, which in free space turns into integration over emission angles $d^{2} q=$ $2 \pi(\omega / c)^{2} \sin \Theta d \Theta$. In the ultrarelativistic limit most of the forward emission is into a cone of opening angle $\Delta \Theta=$ $2 / \gamma$ [with wide bandwidth emission, Eq. (7), in the range between $\omega_{r 0} / 2$ to $\left.\omega_{r 0}\right]$. Therefore an alternative way to obtain a crude estimate of the total forward emission is to multiply the expression for the number of coherent photons (31) by a factor $\frac{1}{4}(\Delta \Theta)^{2} /\left(\Delta \Theta_{\mathrm{coh}}^{2}\right)=L f_{s 0}^{\prime} / 4 \gamma$ :

$$
\left(N_{\mathrm{ph}}\right)_{\mathrm{for}} \cong \frac{2 \pi}{\hbar} \sqrt{\frac{\mu_{0}}{\varepsilon_{0}}} \frac{\mu_{s}^{2} f_{s 0}^{\prime 3} L}{c^{5} \gamma}\langle\cdots\rangle_{\perp s} .
$$

This expression matches well the expression for forward photon emission, derived in Eq. (B5) as half the total number of photons emitted by a magnetic dipole in its rest frame during a finite emission time equal to the transit time $L^{\prime} / v \cong L^{\prime} / c$, when traversing through a LorenzFitzgerald contracted magnet of length $L^{\prime}=L / \gamma$. Evidently the simpler calculation of the total number of emitted photons in the electrons rest frame is valid for the moving dipole case, because this parameter is a Lorenz invariant.

If the particles enter the magnet at random initial times $t_{0 j}$ or random precession phases $\varphi_{s 0 j}$, the mixed terms in $\langle\cdots\rangle_{\perp s}$ cancel out and consequently, within a classical model, the statistical form factor to be substituted in (32) is $\langle\cdots\rangle_{\perp s}=\frac{2}{3} N$ (B7). However, as indicated earlier, a classical analysis is not valid for the calculation of spontaneous emission. Taking the spontaneous emission limit in the quantum calculation of superradiance following Dicke [8] (see Appendix A), provides the enhanced value of the quantum spontaneous emission photon number. Further consideration of the different dependence on the initial spin polarization angle $\Psi_{i}$, namely $\left\langle 1+\cos \Psi_{i}\right\rangle$ instead of $\left\langle\sin ^{2} \Psi_{i}\right\rangle$, produces a modified expression (B6) to be used for the statistical form factor in (31) and (32) for a random electron beam:

$$
\langle\cdots\rangle_{s}^{\text {quant }}=2 N .
$$

We now consider the case of superradiance [10]. In this case the electron beam is tightly bunched: $\omega\left\langle\left(t_{0 j}-\right.\right.$ $\left.\left.t_{0}\right)^{2}\right\rangle^{1 / 2} \ll 2 \pi$, and all particles have the same initial precession phase $\left\langle\left(\varphi_{0 j}-\varphi_{0}\right)^{2}\right\rangle^{1 / 2} \ll 2 \pi$. Therefore their FESFER emissions add up in phase (superradiant emission [10]), and consequently $\langle\cdots\rangle_{\perp s}=N^{2}$. In practice the electron beam is partially polarized: $N_{\uparrow}$ electrons are emitted from the photocathode at "spin up" pure quantum state, $N_{\downarrow}$ at "spin down," and $N_{r}$ - at random spin orientation. After the $\pi / 2$ magnet section, the corresponding first two groups of pure state electrons wind up as two transverse-spin giant magnetic dipoles $\left|\boldsymbol{\mu}_{\uparrow}\right|=N_{\uparrow} \mu_{s},\left|\boldsymbol{\mu}_{\downarrow}\right|=N_{\downarrow} \mu_{s}$ of opposite orientation: $\varphi_{s 0 \uparrow}=\varphi_{s 0 \downarrow}-\pi$, and then

$$
\langle\cdots\rangle_{\perp s}=N^{2}\left(P_{\uparrow}-P_{\downarrow}\right)^{2},
$$

where $P_{\uparrow}=N_{\uparrow} / N, P_{\downarrow}=N_{\downarrow} / N$, and $P_{r}=N_{r} / N$.

Equations (31) and (32) are, respectively, the expressions for coherent and total superradiant FESFER. The classical superradiant expressions are legitimate: as discussed in the appendices, when $N\left(P_{\uparrow}-P_{\downarrow}\right) \gg 1$, the emission of a photon hardly changes the orientation of the giant magnetic dipole [8] that continues to precess as a classical top. It is therefore correct to state, up to a small numerical factor, that when conditions for superradiant emission are satisfied, the FESFER emission will be enhanced by a factor $\approx N\left(P_{\uparrow}-P_{\downarrow}\right)^{2}$. 


\section{OBSERVATION OF SUPERRADIANT FESFER AND CRE}

Magnetic dipole emission is much weaker than electric emission. For this reason the spontaneous FESFER power is miniscule. Taking an example of $B_{0}=10 \mathrm{~T}, L=1 \mathrm{~m}$, $\gamma=10\left(\lambda_{r 0}=8.6 \mu \mathrm{m}\right)$, high electron bunch charge of $q=1 \mathrm{nC}\left(N=6.25 \times 10^{9}\right)$ and bunch repetition rate of $1 \mathrm{GHz}$ we obtain for the coherent and total spontaneous photon emissions respectively (31)-(33): $\left(N_{\mathrm{ph}}\right)_{\mathrm{coh}}^{\mathrm{sp}}=3.6 \times$ $10^{-8} \mathrm{ph} /$ bunch $, \quad d / d t\left(N_{\mathrm{ph}}\right)_{\mathrm{coh}}^{\mathrm{sp}}=36 \mathrm{ph} / \mathrm{s}, \quad\left(N_{\mathrm{ph}}\right)_{\text {for }}^{\mathrm{sp}}=$ $2.2 \times 10^{-5} \mathrm{ph} /$ bunch, $d / d t\left(N_{\mathrm{ph}}\right)_{\text {for }}^{\mathrm{sp}}=2.2 \times 10^{4} \mathrm{ph} / \mathrm{s}$.

The FESFER emission will be enhanced by a big factor $X 3 N / 2$, if the electrons would emit superradiantly. However, this requires that the electron bunch duration will be short relative to the radiation period $f_{r 0} t_{b}<1$. With the present technological state of the art, the available bunch duration is $t_{b}=0.1-1 \mathrm{pS}$, depending on the acceleration energy; therefore we consider a low FESFER frequency example of $f_{r 0}=1 \mathrm{THz} \quad\left(\lambda_{r 0}=\right.$ $300 \mu \mathrm{m})$, which can be attained with $B_{0}=0.5 \mathrm{~T}, \gamma=6$. Setting now in (31) and (32) $\langle\cdots\rangle_{\perp s}=N^{2}$, one obtains for the same beam parameters as in the previous example: $\quad\left(N_{\mathrm{ph}}\right)_{\mathrm{coh}}^{\mathrm{SR}}=0.19 \mathrm{ph} /$ bunch,$\quad d / d t\left(N_{\mathrm{ph}}\right)_{\mathrm{coh}}^{\mathrm{SR}}=$ $1.9 \times 10^{7} \mathrm{ph} / \mathrm{s},\left(N_{\mathrm{ph}}\right)_{\mathrm{for}}^{\mathrm{SR}}=0.56 \mathrm{ph} /$ bunch, $d / d t\left(N_{\mathrm{ph}}\right)_{\mathrm{for}}^{S \mathrm{SR}}=$ $5.6 \times 10^{8} \mathrm{ph} / \mathrm{s}$.

Some enhancement of the coherent spontaneous and superradiant emission can be obtained if the emission takes place in a waveguide of minimal cross-section area $A_{e m}$ and not in free space. This reduces the diffraction effect at long wavelengths and also enables wide coherentemission linewidth $(\Delta \omega)_{\text {coh }}$ near "zero-slippage" conditions [11]. Further substantial enhancement of FESFER emission will be expected if techniques for periodic $\mathrm{THz}$ frequency bunching of the electron beam will be further developed [26].

The calculated flux of FESFER photons emission may be detectable, especially with SR enhancement. However the real obstacle for direct observation of FESFER is the concurrent occurrence, at higher emission rate of cyclotron-resonance-emission (CRE) [27] by electrons that enter the axial magnetic field section with any transverse velocity $\beta_{\perp j}$. Direct calculation of $\Delta \breve{W}_{q j}^{e}$ [Eq. (24)] for an electron entering the uniform axial magnetic field section at time $t_{0 j}$, with initial gyration phase $\varphi_{c 0 j}$ is

$$
\Delta \breve{W}_{q j}^{e}=\left|\Delta \breve{W}_{q}^{e}\right| e^{i\left(\omega_{c 0} t_{0 j}+\varphi_{c 0 j}\right)}
$$

where $\omega_{c o}=\omega_{c 0}^{\prime} / \gamma$

$$
\omega_{c 0}^{\prime}=\frac{e}{m} B_{0}
$$

$$
\begin{gathered}
\left|\Delta \breve{W}_{q}^{e}\right|^{2}=\frac{1}{2}\left(\frac{e L}{\beta}\left|\mathcal{E}_{q}\left(x_{e}, y_{e}\right)\right|\right)^{2} \sin c^{2}\left(\theta_{c} L / 2\right), q \\
\theta_{c}=\frac{\omega-\omega_{c 0}^{\prime} / \gamma}{v_{z}}-k_{z} .
\end{gathered}
$$

The magnetic and electric work functions (28) and (37) are related by simple proportion. For $\Theta \cong 0, \theta_{s}=0, \theta_{c}=$ 0 :

$$
\alpha=\frac{\left|\Delta \breve{W}_{q}^{m}\right|}{\left|\Delta \breve{W}_{q}^{e}\right|}=\frac{\hbar \omega_{c 0}^{\prime}}{2 \gamma m c^{2}} \ll 1 .
$$

Including both terms in the first Eq. (26) one can write

$$
\begin{aligned}
\frac{d W_{q}}{d \omega}= & \frac{1}{8 \pi \mathcal{P}_{q}}\left|\Delta \breve{W}_{q}^{e}\right|_{\max }^{2}\langle| \sum_{j} e^{i \omega t_{0 j}\left[\beta_{\perp j} e^{i \varphi_{c 0 j}+i \theta_{c} L / 2}\right.} \\
& \times \sin c\left(\theta_{c} L / 2\right)+\alpha \sin \Psi_{j} e^{i \varphi_{s 0 j}+i \theta_{s} L / 2} \\
& \left.\left.\times \sin c\left(\theta_{s} L / 2\right)\right]\left.\right|^{2}\right\rangle,
\end{aligned}
$$

where $\left|\Delta \breve{W}_{q}^{e}\right|_{\max }=(e L / \sqrt{2} \beta)\left|\tilde{\mathcal{E}}_{q \perp}^{*} \cdot \hat{\mathbf{e}}_{+}\right|$.

The CRE frequency [corresponding to $\theta_{c}\left(\omega_{r}\right)=0$ ] is given by (7) with $\omega_{c 0}^{\prime}$ substituting $\omega_{s 0}^{\prime}$. Unfortunately the difference between the FESFER and CRE frequencies

$$
\frac{\delta \omega}{\omega_{r}}=\frac{\omega_{s 0}^{\prime}-\omega_{c 0}^{\prime}}{\omega_{c 0}^{\prime}}=\left(\frac{g}{2}-1\right)=1.16 \times 10^{-3}
$$

is very small, and for practical magnet lengths $L$, is smaller than the emission frequency linewidth (30). Consequently it is hard to separate the emission lines (28) and (37) by frequency filtering.

If the CRE and FESFER wave phases are uncorrelated, the mixed CRE/FESFER term resulting from squaring the brackets in (40) vanishes, and it produces in addition to (27) an expression for the CRE spectral power:

$$
\frac{d W_{q}^{e}}{d \omega}=\frac{1}{8 \pi \mathcal{P}_{q}}\left|\Delta \breve{W}_{q}^{e}\right|^{2}\left\langle\left|\sum_{j=1}^{N} e^{i \omega_{c 0} t_{0 j}+i \varphi_{c 0 j}} \beta_{\perp j}\right|^{2}\right\rangle_{\perp c} .
$$

The ratio of FESFER (27) to CRE (42) emissions can be found using (39):

$$
\frac{d W_{q}^{m} / d \omega}{d W_{q}^{e} / d \omega}=\alpha^{2} \frac{\langle\cdots\rangle_{\perp s}}{\langle\cdots\rangle_{\perp c}} \ll 1 .
$$

The first factor $\alpha^{2}\left(\alpha \equiv \hbar \omega_{c 0}^{\prime} / 2 \gamma m c^{2}\right)$ in (43) is very small, but the ratio can be substantially enhanced if the FESFER is superradiant $\left(\left\langle\left(\varphi_{s 0 j}-\varphi_{s 0}\right)^{2}\right\rangle^{1 / 2}, \omega_{r} t_{b} \ll 2 \pi\right)$ and the CRE is spontaneous (namely, the entrance gyration phases $\varphi_{c 0 j}$ are random). In this case $\langle\cdots\rangle_{\perp s} /\langle\cdots\rangle_{\perp c}=$ $N /\left\langle\beta_{\perp}^{2}\right\rangle$. Even with this large factor, the ratio (43) is quite 
small. For the parameters of the last example and an optimistic assumption $\left\langle\beta_{\perp}^{2}\right\rangle^{1 / 2}=10^{-4}$, this ratio is equal to $2.2 \times 10^{-3}$. These conditions would be hard to achieve, because they require accurate alignment of the $e$-beam center with the magnetic axis or careful scanning of the beam to minimize steering angle error. Otherwise $\varphi_{c 0 j}$ cannot be considered random, and consequently also the CRE will be superradiant and proportional to $N^{2}$. If good alignment of the beam can be attained, then increasing the factor (43) beyond 1 is still difficult, but not fundamentally impossible. It requires predominantly an advance in the $e$-beam technology (higher charge per bunch, shorter bunches and smaller emittance) which would permit higher $N$ and $\omega_{r c 0}^{\prime}$ and smaller $\left\langle\beta_{\perp}^{2}\right\rangle$ values in (43).

One can consider an alternative way for measuring FESFER that circumvents the difficulty of the CRE background, and turns it into a helpful means. The mixed term (magnetic-electric emission) which results from squaring (40) [second term in the second equality of Eq. (26)], being proportional to $\alpha$, is much larger than the FESFER term, which is proportional to $\alpha^{2}$ (43). If one operates in conditions where both the FESFER and CRE emissions are superradiant, namely, $\omega_{r} t_{b},\left\langle\left(\varphi_{s 0 j}-\varphi_{s 0}\right)^{2}\right\rangle^{1 / 2},\left\langle\left(\varphi_{c 0 j}-\right.\right.$ $\left.\left.\varphi_{c 0}\right)^{2}\right\rangle^{1 / 2} \ll 2 \pi$, then from (40):

$$
\begin{aligned}
\frac{d W_{q}}{d \omega}= & \frac{1}{8 \pi \mathcal{P}_{q}}\left|\Delta \breve{W}_{q}^{e}\right|_{\max }^{2} N^{2}\left\{\beta_{\perp 0}^{2} \sin c^{2}\left(\theta_{c} L / 2\right)\right. \\
& +2 \alpha \beta_{\perp 0}\left(P_{\uparrow}-P_{\downarrow}\right) \sin c\left(\theta_{c} L / 2\right) \sin c\left(\theta_{s} L / 2\right) \\
& \times \cos \left[(g / 2-1) \omega_{c o}^{\prime} L / \gamma v+\varphi_{c o}-\varphi_{s 0}\right] \\
& \left.+\alpha^{2} \operatorname{sinc}^{2}\left(\theta_{s} L / 2\right)\right\} .
\end{aligned}
$$

The superradiant FESFER (third term) is now negligible relative to the superradiant CRE (first term), but one may be able to observe the combined FESFER/CRE (second term) on top of the CRE background by reversing its sign from pulse to pulse (e.g., by linear transverse scanning of the beam which changes $\varphi_{c 0}$ by $\pi$ ) and averaging over many bunches. The maximum relative change in power is then $4 \alpha / \beta_{\perp 0}$. For the parameters of the previous example $f_{c 0}^{\prime}=88 \mathrm{GHz}, \gamma=6$, and $\beta_{\perp 0}=1 \mathrm{mrad}$, this corresponds to a relative change of $2.5 \times 10^{-7}$. The quantum mechanical picture given in Appendix A portrays the effect of correlation between the polarized spins and the phase correlated cyclotron orbitals as analogous to a big atom with controllable $L-S$ coupling.

Probably a better way of taking advantage of the superradiant CRE emission is to coherently detect the beat wave between its coherent intense field and the coherent FESFER emission. Since the CRE is by nature synchronous with the FESFER and coherently related to it (as long as $\omega t_{b}<2 \pi$ ), it can serve as a local oscillator in an heterodyne detection scheme of the FESFER radiation (in this case one may even desire to enhance the CRE emission by slight beam angular deflection so that $\left.\langle\cdots\rangle_{\perp c}=\beta_{\perp 0}^{2} N^{2}\right)$. To explore this possibility it is necessary to transform (23) to the time domain. Following [28] we obtain

$$
\begin{aligned}
\mathbf{E}\left(\mathbf{r}_{\perp}, L, t\right) \propto & N\left|\tilde{\mathcal{E}}_{q+}\left(\mathbf{r}_{\perp}\right)\right| \operatorname{Re}\left\{\left[\beta_{\perp 0} e^{i \omega_{r c 0}\left(t-t_{0}-L / v\right)+i \varphi_{c 0}}\right.\right. \\
& \left.\left.+i \alpha\left(P_{\uparrow}-P_{\downarrow}\right) e^{i \omega_{r 50}\left(t-t_{0}-L / v\right)+i \varphi_{s 0}}\right] \hat{\mathbf{e}}_{+}\right\} \\
& \cdot f\left(t-t_{0}-L / v\right),
\end{aligned}
$$

where $f(t) \cong \operatorname{rect}\left(t / t_{\mathrm{sl}}\right)$ is the wave packet envelop function and $t_{\mathrm{sl}}=2 \pi /(\Delta \omega)$ is the radiation slippage time, which for a short bunch is equal to the radiation pulse duration. When this field is detected by a square-law detector, the measured signal will be proportional to

$$
\begin{aligned}
\left|\mathbf{E}\left(\mathbf{r}_{\perp}, L, t\right)\right|^{2} \propto & N^{2}\left|\tilde{\mathcal{E}}_{q+}\left(r_{\perp}\right)\right|^{2}\left\{\beta_{\perp 0}^{2}+\alpha \beta_{\perp 0}\left(P_{\uparrow}-P_{\downarrow}\right)\right. \\
& \left.\times \sin \left[\delta \omega\left(t-t_{0}-L / v\right)+\varphi_{c 0}-\varphi_{s 0}\right]\right\} \\
& \times f^{2}\left(t-t_{0}-L / v\right) .
\end{aligned}
$$

Since for practical parameters, $\delta \omega t_{\mathrm{sl}}=2 \pi \delta \omega / \Delta \omega$ is smaller than $2 \pi$ [see (30) and (41)] it may be hard to have CRE-FESFER beat oscillation over a number of periods in a single pulse. Yet, similar waveforms are expected in each pulse, if good beam stability can be maintained, and therefore the beat signal [second term in (46)] can be distinguished from the first term after processing and averaging over many pulses. For example, consider adjusting the initial cyclotron gyration and spin resonance precession phases to be in phase: $\varphi_{c 0}=\varphi_{s 0}$ or out of phase: $\varphi_{c 0}=$ $\varphi_{s 0}-\pi$. If $\delta \omega t_{\mathrm{sl}} \ll 2 \pi$, the first order Taylor expansion of the second term in (42) is $\pm \alpha \beta_{\perp 0}\left(P_{\uparrow}-P_{\downarrow}\right) \delta \omega\left(t-t_{0}-\right.$ $L / v)$. Differentiation of the signal will null the contribution of the first term and leave the second term. Signal averaging over many pulses, along with modulation of the Cyclotron or Spin phase or amplitude, and corresponding correlated processing, can reveal the FESFER/ CRE beat signal out of random noise.

\section{CONCLUSION}

A complete formulation, in the framework of a classical model, was presented for evaluating the characteristics of electron spin resonant radiative emission from a polarized free electrons beam (FESFER) and for changing (modulating) its spin state (FESMER).

FESFER emission is weak, but its observation is not fundamentally prohibited. It can be substantially enhanced at superradiance emission conditions. Achievement of increased charge per bunch of polarized particles will enhance the superradiant emission quadratically. Emerging techniques for periodic e-beam bunching at $\mathrm{THz}$ frequencies [29] may provide enhancement of the FESFER photon emission rate. A promising method for detecting and measuring FESFER is by heterodyne detection of its beat with the concurrent CRE radiation, which slightly deviates in frequency because of the gyromagnetic factor $g$. The 
FESFER measurement can be used for noninvasive diagnosis of the spin polarization state of polarized electron beams.

The FESMER process makes it possible to change the polarization state of a polarized $e$-beam by applying on it an intense EM radiation wave at ESR conditions. This can be used to change the axial spin polarization of electron beam bunches emitted from the $e$-gun and turn it to transverse-spin state, which is optimal for FESFER emission. It can also be useful for fast electronic modulation or switching of the electron spin-state of a polarized $e$-beam in order to attain sensitive control of spin-dependent electron scattering experiments. These are used at high beam energies for fundamental nuclear structure studies (like parity violation experiments, charge and magnetic polarization distribution within the proton and the dynamics governing its quark wave function distribution), and also at low energy experiments, where polarized e-beam microscopes (e.g. SPLEEM [18]) are used for solid state magnetic material studies. We also suggest that the new formulation can be relevant in connection to the design of the International Linear Collider [21], which requires schemes for spin-polarizing electron/positron beams and controlling and evaluating their spin state.

Finally, it is pointed out that the presented classical analysis has definite limitations when considering incoherent spontaneous emission from a single electron. A quantum mechanical analysis of this problem, with more accurate account of the spontaneous emission limit and the relativistic Thomas precession effect, is called for [22]. This, however, is not expected to change dramatically the main conclusions of this paper: the possibility to modulate a polarized free electron beam with a radiation wave at ESR conditions (FESMER); the significant enhancement of the FESFER emission by a polarized beam at superradiant ESR conditions; the feasibility of a coherent detection scheme for observing the superradiant ESR FESFER radiation in the background of the concurrent cyclotron resonance emission from the beam.

\section{ACKNOWLEDGMENTS}

I would like to thank A. Yariv, G. Alexander, M. Poelker, and B. Colson for helpful discussions, and S. Mane for communicating and advising me regarding the quantum calculation of spontaneous spin-flip emission. This work was done in the framework of the FEL Knowledge Center for Radiation Sources and Applications, sponsored by the Israeli Ministry of Science.

\section{APPENDIX A: TRANSITION FROM QUANTUM TO CLASSICAL LIMIT IN THE SUPERRADIANT EMISSION REGIME}

Transition from quantum description to the classical limit is always expected when large quantum numbers are involved. The validity of the classical expression for the superradiance case can be explained by the fact that the orientation of the "giant dipole" hardly changes with the emission of a photon, and thus it precesses as a classical top. I will elaborate this argument using Dicke's formulation [8]. Even though he applied his quantum mechanical analysis to an assembly of two-quantum-levels molecular electric dipoles, it applies as well (as he points out) to spins.

Following Dicke's formulation, the eigenstates and quantum numbers of an assembly of dipoles in a spatial region smaller than a radiation wavelength (in which the dipole approximation applies for the assembly), are defined by

$$
\begin{gathered}
\mathcal{H} \Psi_{g m r}=\left(\mathcal{H}_{0}+R_{z} \Delta E\right) \Psi_{g m r}=\left(E_{g}+m \Delta E\right) \Psi_{g m r} \\
R^{2} \Psi_{g m r}=r(r+1) \Psi_{g m r},
\end{gathered}
$$

where $\Delta E=\hbar \omega$ is the two levels energy spacing and $E_{g}$ is the energy associated with the other degrees of freedom. In our case (say we are in the electron rest frame) $\Delta E=\hbar \omega_{s 0}^{\prime}$

$$
R_{k}=\sum_{j=1}^{N} R_{j k} \quad(k=x, y, z)
$$

$\left(R_{j k}\right.$ are the Pauli matrices)

$$
R^{2}=R_{x}^{2}+R_{y}^{2}+R_{z}^{2}
$$

The maximal value of $m$ and $r$ is

$$
r=m=\frac{1}{2} N
$$

Calculating the matrix element of the radiative interaction Hamiltonian

$$
\begin{aligned}
& \left\langle g, r, m\left|\hat{\mathbf{e}}_{x} R_{x}+\hat{\mathbf{e}}_{y} R_{y}\right| g, r, m \mp 1\right\rangle \\
& \quad=\frac{1}{2}\left(\hat{\mathbf{e}}_{x} \pm i \hat{\mathbf{e}}_{y}\right)[(r \pm m)(r \mp m+1)]^{1 / 2},
\end{aligned}
$$

Dicke arrives to the following expression for spontaneous/ superradiant emission:

$$
I=I_{0}(r+m)(r-m+1),
$$

where $I_{0}$ is the spontaneous emission from a single dipole $\left(r=m=\frac{1}{2}\right.$ ). When $r=m=\frac{1}{2} N$ [Eq. (A5) - all spins are aligned, all in the axial direction], one gets $I=N I_{0}$, the same as would be limited by $N$ independent spins (contrary to the classical model results that predict zero emission in this case).

If $r=n=N / 2$ (all dipoles are aligned) but $m$ is arbitrary, then

$$
I=I_{0} \frac{1}{4} N^{2}\left(1+\frac{m}{n}\right)\left(1-\frac{m+1}{n}\right) .
$$

Unless $n-m \ll 1$ (which for $n=N / 2 \gg 1$ happens only for nearly pure axial polarization), then 


$$
I=I_{0} \frac{1}{4} N^{2}\left[1-\left(\frac{m}{n}\right)^{2}\right] .
$$

Define $\cos \Psi=m / n$, then

$$
I=\frac{1}{4} I_{0} N^{2} \sin ^{2} \Psi
$$

and the scaling with $\Psi$ is expected in a classical model (27).

It is interesting to extend the quantum point of view also for better understanding of the CRM/FESFER radiation interference effect discussed in Sec. V [Eq. (40)].

The analysis above tempts to regard the electron bunch like an atom with many electrons where the resultant spin operator is

$$
\mathbf{S}=\sum \mathbf{S}_{j}
$$

Contrary to an atom there is no central confining force of the nucleus, and therefore no $L-S$ coupling. We only have Landau levels for each electron, nearly degenerate with their spin levels. If the dimensions of the bunch and the cyclotron radius are smaller than a wavelength (in its rest frame) then the entire bunch can radiate as a single dipole similarly to the shell electrons in an atom in the dipole approximation. Contrary to the atom, the bunch from a photocathode e-gun is not a steady state solution of a problem, and there are no central force and significant electron mutual interaction forces to arrange the electrons' spins and orbitals configuration in particular patterns during the bunch flight through the magnetic field section. Furthermore, because of the low density of the electrons we are not limited by the Fermi exclusion principle. The configuration depends here only on the initial conditions, namely the cathode material and the electron optics. Thus, we have the freedom to create correlated spin and orbital configurations at will (namely polarized electrons with desirable spins orientation and a correlated configuration of cyclotron orbitals in the axial magnetic section). Stretching the analogy to an atom further, we can view the combined superradiant CRM/FESFER emission, which is discussed in Sec. V classically, as a version of Zeeman effect transitions. Define the total angular momentum of the bunch:

$$
\mathbf{J}=\mathbf{L}+\mathbf{S}
$$

where

$$
\mathbf{L}=\sum_{i=1}^{N} \mathbf{L}_{i}, \quad \mathbf{S}=\sum_{i=1}^{N} \mathbf{S}_{i}
$$

Not only the spin states, but also the orbital states, are quantized, and the unperturbed Hamiltonian should be

$$
\mathcal{H}=\hbar \omega_{c} L_{z}+2 \hbar \omega_{s} S_{z}
$$

The total angular momentum $\mathbf{J}$ (A12) should replace $\mathbf{S}$
(A12), and instead of $r$ there will be in (A7) the total angular quantum number $j$, where $j(j+1)$ is the eigenvalue of $J^{2}$. When the matrix element of the radiative interaction perturbation Hamiltonian is calculated, there will be correspondence between the terms originating from $\mathbf{L}$ and $\mathbf{S}$ in (A12) and the terms of Eq. (44) $\left(\theta_{c} \approx \theta_{s}\right)$. Scanning the electron beam angle across the magnetic axis (reversing the phase: $\varphi_{c 0} \rightarrow \varphi_{c 0}+\pi$ ), as suggested subsequent to Eq. (44), would be equivalent to preparing an "atom" with $\mathbf{L}$ and $\mathbf{S}$ at an enhanced or reduced total momentum $(\mathbf{J}=\mathbf{L}+\mathbf{S})$.

\section{APPENDIX B: PHOTON EMISSION FROM AN OSCILLATING MAGNETIC DIPOLE ANTENNA}

It is instructive to compare the expression calculated for the total number of photon emissions from a classical magnetic dipole antenna to the expressions for the total forward FESFER photon emission, calculated in this paper for the superradiant and spontaneous emission cases.

The standard expression for the total power emission by a dipole antenna,

$$
\mathbf{m}=m_{0}\left\lfloor\hat{\mathbf{e}}_{x} \cos \left(\omega t+\varphi_{0}\right)+\hat{\mathbf{e}}_{y} \sin \left(\omega t+\varphi_{0}\right)\right\rfloor
$$

is

$$
P=\frac{1}{6 \pi \varepsilon_{0}} \frac{m_{0}^{2} \omega^{4}}{c^{5}} .
$$

The number of photons emission in a finite radiation time $T$ can be then calculated straightforwardly as

$$
\left(N_{\mathrm{ph}}\right)_{\mathrm{tot}}=\frac{P T}{\hbar \omega}=\frac{4 \pi^{2}}{3} \sqrt{\frac{\mu_{0}}{\varepsilon_{0}}} \frac{m_{0}^{2} f^{3}}{\hbar c^{4}} T .
$$

Since the number of photons is a Lorenz invariant, we can use this expression in the rest frame of the electron beam in order to compare to the expressions calculated before for the FESFER photons number. It is possible then to set $\omega=$ $2 \pi f_{s 0}^{\prime}, m_{0}=N \mu_{s}^{\prime}$, and $T=L / \gamma c$, where $T$ is the interaction time in the electron rest frame during the traversal through a Lorenz contracted magnet of length $L / \gamma$ :

$$
\left(N_{\mathrm{ph}}\right)_{\mathrm{tot}}=\frac{4 \pi^{2}}{3} \sqrt{\frac{\mu_{0}}{\varepsilon_{0}}} \frac{\mu_{s}^{2} f_{s}^{\prime 3}}{\hbar c^{5}} \frac{L}{\gamma} N^{2} .
$$

Note, however, that this is the number of electrons emitted in all directions. The Doppler upshifted photons emitted in the forward direction within a cone of opening angle $2 / \gamma$ (in the lab frame) are only half the total number (B4).

Equation (B4) can be employed directly for the case of superradiant emission $(N \gg 1)$, where the classical expressions are valid:

$$
\left(N_{\mathrm{ph}}\right)_{\text {for }}=\frac{2 \pi^{2}}{3} \sqrt{\frac{\mu_{0}}{\varepsilon_{0}}} \frac{\mu_{s}^{2} f_{s}^{\prime 3}}{\hbar c^{5}} \frac{L}{\gamma}\langle\cdots\rangle_{\perp s} .
$$

Here $|\langle\cdots\rangle|_{\perp s}=N^{2}$, which is the case of maximum emis- 
sion $\left(\Psi=\pi / 2, P_{\uparrow}-P_{\downarrow}=1\right)$ of the general superradiance form factor

$$
\langle\cdots\rangle_{\perp s}=N^{2}\left(P_{\uparrow}-P_{\downarrow}\right)^{2} \sin ^{2} \Psi .
$$

In a simplistic employment of (B4) to calculation of spontaneous emission, one would set $N=1$ and sum up the contribution from different randomly polarized electrons. Equation (B5) would then be used with a factor

$$
\langle\cdots\rangle_{\perp s}=\left\langle\sum_{j=1}^{N} \sin ^{2} \Psi_{j}\right\rangle=\frac{2}{3} N
$$

This expression is not really valid, since in the spontaneous emission limit a quantum analysis results in somewhat different expressions and magnitude of the emission rate. According to (A11), the quantum mechanical total photon emission number per electron is four times the number predicted by the classical expression (B4) $(N=$ 1). Furthermore, this factor is calculated specifically for the case that the spin is initially at the upper quantum state $(m=1 / 2)$. The dependence of the spontaneous emission rate on the initial inclination angle of the spin $\Psi$, relative to the magnetic axis, is not as in the classical expression (B7) but scales like $\left(1+\cos \Psi_{j}\right)$ [22]. Consequently, for randomly oriented spins or for a transversely polarized beam, not satisfying the superradiance condition, the four factor is reduced by half, and finally the quantum-mechanical expression for forward FESFER photon emission by a random electron beam is given by (B5) with

$$
\langle\cdots\rangle_{\perp s}=2 N
$$

(an alternative explanation for the factor $1 / 2$ is that random or transversely polarized single electrons excite states $m \pm$ $1 / 2$ with equal probability half, and only the $m=1 / 2$ states contribute to photon emission).

[1] J. Pierce, Traveling Wave Tubes (Van Nostrand, Princeton, 1950).

[2] A. Gover, "Lasers: Free-Electron Lasers," Encyclopedia of Modern Optics, edited by R. D. Guenther, D. G. Steel, and L. Bayvel (Elsevier, Oxford, 2005).

[3] H. Scovil, G. Feher, and H. Seidel, Phys. Rev. 105, 762 (1957).

[4] A. A. Sokolov and I. M. Ternov, Dokl. Akad. Nauk SSSR 153, 1052 (1963) [Sov. Phys. Dokl. 8, 1203 (1964)].

[5] J. D. Jackson, Rev. Mod. Phys. 48, 417 (1976).

[6] S. A. Belomesthnykh et al., Nucl. Instrum. Methods Phys. Res., Sect. A 227, 173 (1984).

[7] K. Kirsebom, U. Mikkelsen, E. Uggerhoj, K. Elsener, S.
Ballestero, P. Sona, and Z.Z. Vilakazi, Phys. Rev. Lett. 87, 054801 (2001).

[8] R. H. Dicke, Phys. Rev. 93, 99 (1954).

[9] G. L. Carr, M. C. Martin, W. R. McKinney, K. Jordan, and G. Neil, Nature (London) 420, 153 (2002).

[10] A. Gover, Phys. Rev. ST Accel. Beams 8, 030701 (2005).

[11] A. Gover, E. Dyunin, Y. Lurie, Y. Pinhasi, and M. V. Krongauz, Phys. Rev. ST Accel. Beams 8, 030702 (2005).

[12] A. Gover, Phys. Rev. Lett. 96, 124801 (2006). This paper contains some errors and omissions, corrected here: The limits of validity of the classical derivation of spontaneous emission are more clearly expressed, the approximate "classical expression" for the spontaneous FESFER photon emission number is replaced by a slightly enhanced quantum-mechanical expression, and missing reference is given to experimental observations of spontaneous FESFER [7,6].

[13] R. Alley et al., Nucl. Instrum. Methods Phys. Res., Sect. A 365, 1 (1995).

[14] Kenji Abe et al., Phys. Rev. Lett. 84, 5945 (2000).

[15] O. Gayou et al., Phys. Rev. Lett. 88, 092301 (2002).

[16] J. Unguris, A. Seiler, R. J. Celotta, D. T. Pierce, P. D. Johnson, and N. V. Smith, Phys. Rev. Lett. 49, 1047 (1982).

[17] H.P. Oepen and J. Kirschner, Scanning Microsc. 5, 116 (1991).

[18] R. Zdyb and E. Bauer, Phys. Rev. Lett. 88, 166403 (2002).

[19] D. D. Awschalom and J. M. Kikkawa, Phys. Today 52, No. 6, 33 (1999).

[20] A. Yariv, Quantum Electronics (John Wiley, New York, 1988).

[21] Report No. EPP2010, 2005 (http://www.linearcollider. org/cms).

[22] S. R. Mane (private communication). A quantum analysis results in for the spontaneous emission case a modified expresson, in which the scaling with the initial spin orientation is $\left\langle 1+\cos \Psi_{j}\right\rangle$. Also, possible effect of Thomas precession on the spin-flip emission rate needs to be examined elsewhere.

[23] J. Schwinger, Phys. Rev. 73, 416 (1948).

[24] V. Bargmann, L. Michel, and V.L. Telegdi, Phys. Rev. Lett. 2, 435 (1959).

[25] A.A. Barybin and V.A. Demitriev, Modern Electrodynamics and Coupled Modes Theory (Rinton Press, Princeton, NJ, 2002).

[26] J. G. Neumann, J. R. Harris, B. Quinn, and P. G. O'Shea, Rev. Sci. Instrum. 76, 033303 (2005).

[27] D. B. Melrose and K. Russelle, J. Phys. A 35, 135 (2002).

[28] A. Gover, F. V. Hartemann, G. P. LeSage, N. C. Luhmann, R. S. Zhang, and C. Pellegrini, Phys. Rev. Lett. 72, 1192 (1994).

[29] Yen-Chieh Huang, Proceedings of the 2005 ICFA Joint Workshop on Laser-Beam Interactions and Laser \& Plasma Accelerators, Taipei, Taiwan (to be published). 